Pak. J. Agri. Sci., Vol. 55(2), 433-440; 2018

ISSN (Print) 0552-9034, ISSN (Online) 2076-0906

DOI:10.21162/PAKJAS/18.5616

http://www.pakjas.com.pk

\title{
ASSOCIATION OF SIX SELECTED PATHOGENICITY GENES OF Escherichia coli WITH GROSS AND HISTOPATHOLOGICAL LESIONS IN BROILER CHICKENS FROM FIELD CASES
}

\author{
Abdul Wadood Jan', M. Tariq Javed ${ }^{1, *}$, Sameen Qayoom Lone' ${ }^{1}$, Aziz-ur-Rehman', \\ M. Sohaib Aslam ${ }^{1}$ and Ahmad Javed ${ }^{2}$
}

\author{
${ }^{1}$ Department of Pathology, Faculty of Veterinary Science, University of Agriculture Faisalabad-38040, Pakistan; \\ ${ }^{2}$ Cardiac Centre, District Head Quarter Hospital, Faisalabad, Pakistan. \\ Corresponding author's e-mail: javedmt @outlook.com
}

\begin{abstract}
Escherichia coli infection is of high significance in chicken, especially in broilers. It produces lesions such as air sacculitis, peritonitis, peri-hepatitis, pericarditis, osteomyelitis, synovitis and/or omphalitis. The present study was conducted E. coli infected broiler chickens from field in and around Faisalabad. The main objective of the study was to detect different pathogenicity genes (fimC, tsh, iucD, papC, fyuA, irp2) of E. coli and associated lesions in broilers. The birds showing suspected postmortem findings for E. coli were further investigated for histopathological studies and culture isolation. The observations revealed that E.Coli infection was found in $84 \%$ of open house farm and $16 \%$ controlled houses farms. The genes detected at higher prevalence were fim $\mathrm{C}$ having $92 \%$ prevalence, followed by $t s h 80 \%$, iucD $72 \%$, fyuA $60 \%$, papC $48 \%$ and $\operatorname{irp} 232 \%$, respectively. A total of 17 strains were identified on the basis of presence or absence of these six genes. These results also indicate the involvement of certain genes more than others in producing severe changes in different body tissues as in more than $50 \%$ cases, the genes including fim $C$, tsh and iucD were detected.
\end{abstract}

Keywords: broilers, E. coli, gross pathology, histopathology, pathogenicity genes

\section{INTRODUCTION}

The poultry meat produced in Pakistan is $28 \%$ of the total meat production in the country. There is a wide range of infectious diseases which affect the productive performance of the birds (Ahmad et al., 2012). Among infectious diseases, bacterial diseases are of high significance. These causes significant losses in the poultry industry in terms of high morbidity, mortality, decreased egg production and hatchability (Alexander, 2000). Among the bacterial diseases, colibacillosis is one of the important disease of poultry, producing lesions in different systems of the body (Rodrigue et al., 2005) and one of the main causes of economic losses in the broiler industry (Biswas et al., 2006). The disease is caused by avian pathogenic Escherichia coli (APEC) (Delicato et al., 2003) and the infections are widely reported in the poultry sector across the globe every year with mortality ranging from $5-55 \%$ (Gomes et al., 2005). This E. coli is normally present in the intestinal tract of the birds, but 10 to $15 \%$ strains have the potential to cause the disease. This disease mostly occurs secondary to the primary infection like viral infections or mycoplosmosis and poor management. The main clinical signs include depression, anorexia, dullness, high body temperature, difficulty in breathing and whistling (Anjum, 1997). It produces lesions such as air sacculitis, peritonitis, perihepatitis, pericarditis, osteomyelitis, synovitis or omphalitis (Kumar et al., 2013).
There are different virulence factors which lead to the development of lesions, especially in body tissues other than intestine. The described virulence factors for APEC, so far, are hemolysin, adhesins, iron-acquisition systems, antibactericidal factors and toxins (Ewers et al., 2004). By using molecular techniques such as PCR, it is now possible to characterize virulence genes (iucD, papC, tsh, fimC, iss and irp2) (Ewers et al., 2004). The fimC and papC are fimbria related virulence genes. The fimC play an important role in the adhesions of bacteria to the respiratory tract of the host and papC for adhesions to the internal organs of the host. The $i u c D$ and irp2 are iron-acquisition system virulence factors which play a role by removal of iron from the host cells and enhances the multiplication and growth of the bacteria. The tsh is a heamolysin virulence factor which causes destruction of the erythrocytes and iss gene plays an important role in the protection of bacteria against the bactericidal activity of the host. The APEC strains have the potential of zoonotic risk (Ewers et al., 2004). Although, a couple of studies have been reported in Pakistan on colibacillosis in broilers, but the application of molecular techniques for the isolation of virulent genes has still not been carried out. So, the present study was planned to detect the virulence genes in APEC in local isolates and to find an association of six selected genes with gross and histopathological lesions in broilers. 


\section{MATERIALS AND METHODS}

The sampling was done from sick and recently dead broilers coming to the Diagnostic laboratory, Department of Pathology, Faculty of Veterinary Science, UAF, District disease Diagnostic Laboratory Faisalabad and Civil Veterinary Hospital Faisalabad. Observations about farm (type of farm and start of treatment on their own) and birds (excreta, appetite, age and weight) were recorded. The postmortem and laboratory findings (Gross and histopathology, culture test and PCR) were recorded.

Collection of samples: The total samples collected $(\mathrm{n}=50)$ were of 25 broilers farms with suspected E. coli infection from Feb 2, 2015 to June 30, 2015. The morbid organs including liver, heart, spleen, intestine, lung, etc. were collected into two different containers for culture isolation and histopathology.

Gross and microscopic studies: Scoring of gross lesions was done on the basis of severity of lesions, i.e., no lesions (0), mild (1), moderate (2) and severe (3). Tissue processing, slides preparation and staining was done by hematoxylin and eosin technique following the method as described earlier (Bancroft and Gamble 2008). The microscopic lesions in different organs were also recorded on the basis of severity as for gross lesions. The mild microscopic lesions were graded on the basis of scant infiltration of inflammatory cells at some places with degenerative and necrotic changes in parenchymal cells in 1-2 microscopic fields at 400X. The moderate lesions were graded on the basis of infiltration of inflammatory cells covering larger (3-4 microscopic fields at 400X) with degenerative and necrotic changes in parenchymal cells. The severe lesions were graded on the basis of infiltration of inflammatory cells expanding to more than 4 microscopic fields at $400 \mathrm{X}$ with degenerative and necrotic changes.

Bacterial Isolation and Identification: Organs collected from the birds were subjected to the culture isolation.
Culturing was carried out on Nutrient agar and MacConkey agar and incubated aerobically at $37^{\circ} \mathrm{C}$ overnight (Tonuet et al., 2011). The growth obtained was shifted to triple sugar iron medium slants and the E. coli was identified by their ability to ferment the sugar lactose. The $E$. coli was further confirmed by colony PCR. The DNA was obtained from bacterial colonies by boiling method by taking 3-4 colonies in $1 \mathrm{ml}$ of distilled water (Mazaheri et al., 2014). Identification of $E$. coli was done by using specific primers (ECO), targeting $16 \mathrm{~S}$ ribosomal DNA of $E$. coli and then six different pathogenicity genes of E. coli (fimC, tsh, iucD, papC, fyuA, irp2) were targeted for amplification using specific primers. The primers selected were from previous studies as indicated below in Table 1.

The PCR was carried out by taking 4ul of template DNA, 18 ul of master mix (Enzynomics), 1.5ul of each primers $(\mathrm{F}+\mathrm{R})$ and $5 \mathrm{u} / \mu 1$ of Taq polymerase. The PCR tubes containing the mixture were placed in thermocycler (Qantarus). The thermal cycling conditions were followed as described by different workers indicated in Table for each gene. The products were run on $1.5 \%$ agarose gel at $90 \mathrm{~V}$ for 45 minutes. The gel was stained with ethidium bromide and visualized by gel documentation system (Biorad).

Ethics statement: Ethical clearance for the study was obtained from the Ethics Review Committee, University of Agriculture Faisalabad. Informed oral consent was obtained from the poultry farm owners at the time of sample collection.

\section{RESULTS}

The observations revealed that $84 \%$ of the farms were open houses, while $16 \%$ were controlled houses on which the $E$. coli problem was reported and confirmed. It was also noted that $56 \%$ of the farmers did not start the treatment on their own. The excreta were abnormal in $76 \%$ cases and appetite in $60 \%$ cases, while in $64 \%$ cases the age of the birds was between 10 and 25 days. The weight of the birds in $60 \%$ cases

Table 1. List of Primers and their sequence with targeted genes.

\begin{tabular}{|c|c|c|c|}
\hline Primer & Primer sequence $(5,-3$ ') & Detected Genes & Reference \\
\hline$F I M C$-f & GGGTAGAAAATGCCGATGGTG & fimC & JanBen et al., 2001 \\
\hline$F I M C$-r & CGTCATTTTGGGGGTAAGTGC & fimC & \\
\hline$P A P C-\mathrm{f}$ & TGATATCACGCAGTCAGTAGC & papC & JanBen et al., 2001 \\
\hline$P A P C-\mathrm{r}$ & CCGGCCATATTCACATAA & papC & \\
\hline$T S H-f$ & ACTATTCTCTGCAGGAAGTC & $T s h$ & JanBen et al., 2001 \\
\hline $\mathrm{TSH}-\mathrm{r}$ & CTTCCGATGTTCTGAACGT & Tsh & \\
\hline FуиA-f & GCGACGGGAAGCGATGACTTA & fуиA & JanBen et al., 2001 \\
\hline Fyu A-r & CGCAGTAGGCACGATGTTGTA & fуиA & \\
\hline HMWP-2-f & AAGGATTCGCTGTTACCGGAC & irp2 & Ewers et al., 2004 \\
\hline HMWP-2-r & TCGTCGGGCAGCGTTTCTTCT & $\operatorname{irp} 2$ & \\
\hline AERA-f & ACAAAAAGTTCTATCGCTTCC & iucD & Ewers et al., 2004 \\
\hline AERA-r & CCTGATCCAGATGATGCTC & iисD & \\
\hline ECO-f & GACCTCGGTTTAGTTCACAGA & 16s rDNA gene & Tonu et al., 2011 \\
\hline ECO-r & CACACGCTGACGCTGACCA & & \\
\hline
\end{tabular}


was below $1 \mathrm{~kg}$. The gross lesions recorded in liver and heart were peri-hepatitis, pericarditis, i.e., fibrinous layer on the surface of the liver and heart as an inflammatory evidence (Fig. 1).

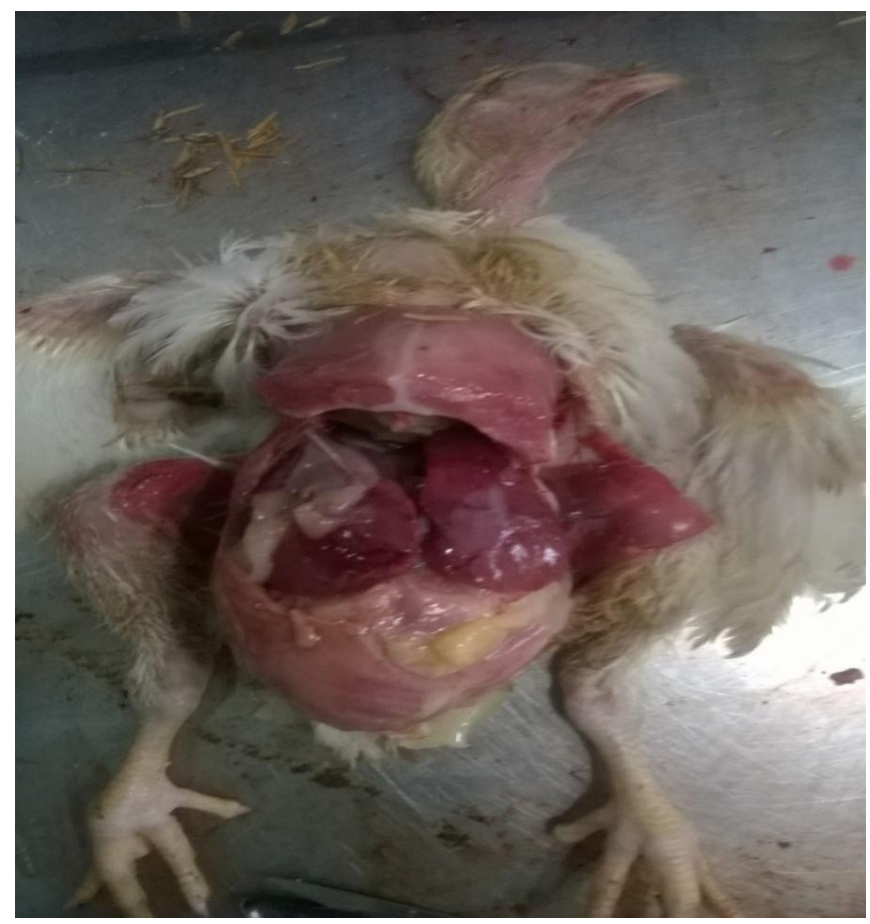

Figure 1. The figure shows typical gross lesions of Colibacillosis, i.e., perihepatitis and pericarditis.

The gross lesions recorded in spleen were enlargement and congestion and in the intestine were congestion and hemorrhages. Lesions were classified as mild, moderate and severe, both grossly and microscopically. The study revealed that liver lesions were present in $100 \%$ cases, out of which $12 \%$ showed mild, $20 \%$ moderate and $68 \%$ severe gross lesions. The lesions in heart were present in $96 \%$ cases, out of which $4 \%$ cases showed mild, $40 \%$ moderate and $52 \%$ severe lesions (Table 2). The lesions in spleen were present in $80 \%$ cases, out of which $44 \%$ showed mild, $12 \%$ moderate and $24 \%$ severe. The lesions in the intestine were present in $96 \%$ cases, out of which $20 \%$ cases showed mild, $48 \%$ moderate and $28 \%$ severe. Gross lesions were also recorded in other organs and those were congested lungs, thickened and cloudy air sacs, congestion in trachea and pale kidneys.

Histopathological lesions observed in liver $r$ were inflammation, congestion and degenerative changes with vacuolation in the cytoplasm of hepatocytes (Fig. 2). The lesions in heart were degenerative changes in the muscles along with infiltration of inflammatory cells (Fig. 3). Degenerative and necrotic changes in the splenic cells with depletion of the nodules were the changes seen in the spleen and disperse increase in lymphocyte population.
Table 2. Results of gross and microscopic lesions observed in four organs with degree of severity have been presented.

\begin{tabular}{|c|c|c|c|}
\hline $\begin{array}{l}\text { Severity of } \\
\text { lesion in } \\
\text { organs }\end{array}$ & $\begin{array}{l}\text { Gross } \\
\text { n }(\%)\end{array}$ & $\begin{array}{c}\text { Microscopic } \\
\text { n }(\%)\end{array}$ & $\begin{array}{c}\text { Difference of } \\
\text { Microscopic lesions } \\
\text { from gross lesions \% }\end{array}$ \\
\hline \multicolumn{4}{|l|}{ Liver } \\
\hline No & $0(0)$ & $0(0)$ & 0 \\
\hline Mild & $6(12)$ & $6(12)$ & 0 \\
\hline Moderate & $10(20)$ & $8(16)$ & -4 \\
\hline Severe & $34(68)$ & $36(72)$ & +4 \\
\hline \multicolumn{4}{|l|}{ Heart } \\
\hline No & $2(4)$ & $2(4)$ & 0 \\
\hline Mild & $2(4)$ & $2(4)$ & 0 \\
\hline Moderate & $20(40)$ & $14(28)$ & -12 \\
\hline Severe & $26(52)$ & $32(64)$ & +12 \\
\hline \multicolumn{4}{|l|}{ Spleen } \\
\hline No & $10(20)$ & $10(20)$ & 0 \\
\hline Mild & $22(44)$ & $12(24)$ & -20 \\
\hline Moderate & $6(12)$ & $16(32)$ & +20 \\
\hline Severe & $12(24)$ & $12(24)$ & 0 \\
\hline \multicolumn{4}{|l|}{ Intestine } \\
\hline No & $2(4)$ & $2(4)$ & 0 \\
\hline Mild & $10(20)$ & $8(16)$ & -4 \\
\hline Moderate & $24(48)$ & $20(40)$ & -8 \\
\hline Severe & $14(28)$ & $20(40)$ & +12 \\
\hline
\end{tabular}

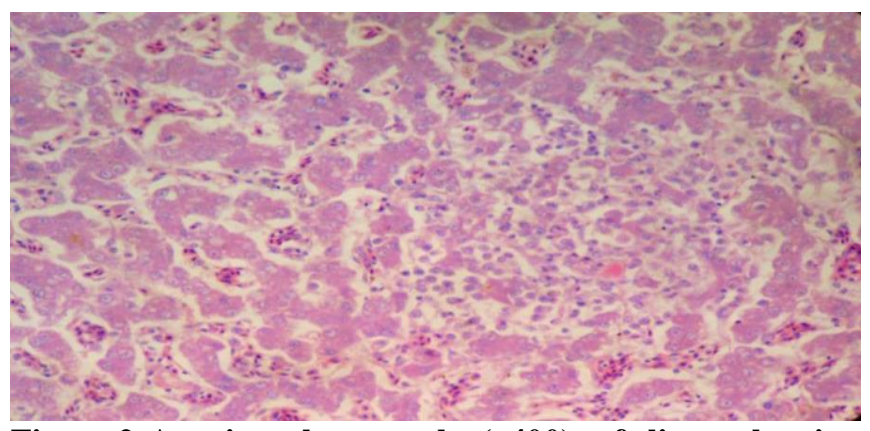

Figure 2. A microphotograph (x400) of liver showing moderate inflammation with degenerative changes in the hepatocytes (vacuoles in the cytoplasm).

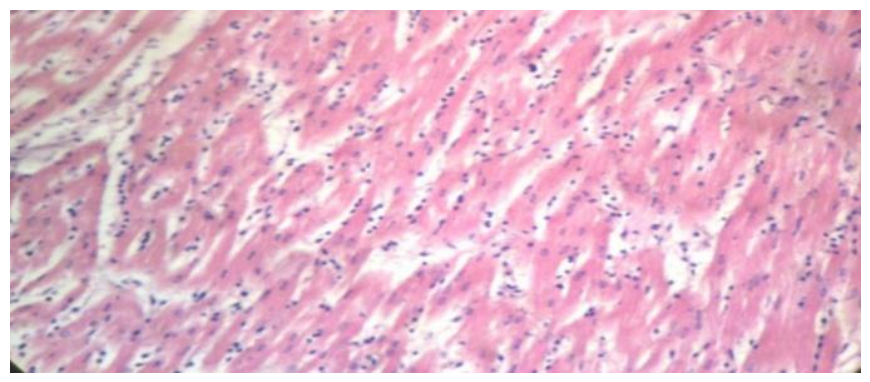

Figure 3. Microphotograph $(\mathbf{x 4 0 0 )}$ of heart muscles showing moderate inflammatory changes in the muscles along with the degenerative changes. 
The changes seen in the intestine were sloughing of epithelium, congestion and accumulation of inflammatory cells in the lamina propria and submucosa.

The histopathological lesions in liver, heart, spleen and intestine were categorized as mild, moderate and severe. In liver, the lesions were mild in $12 \%$ cases, moderate in $16 \%$ cases and severe in $72 \%$ cases. In $4 \%$ cases, no lesions were recorded in the heart, while these were mild in $4 \%$ cases, moderate in $28 \%$ cases and severe in $64 \%$. The lesions in spleen were in following order, i.e., $20 \%$ had no, $24 \%$ had mild, $32 \%$ moderate and $24 \%$ had severe. The lesions in the intestine were not seen in $4 \%$ cases, $16 \%$ cases had mild, $40 \%$ moderate and $40 \%$ had severe.

The growths on MacConkey agar were obtained from all the 50 cases from 25 farms, while on nutrient agar, growths from 46 cases (23 farms) could be obtained. These growths obtained on MacConkey agar were further confirmed by Gram staining, growth characters on TSI and by PCR using E. coli specific primers.

Strains identified: The gene specific PCR carried out on 50 isolates from 25 farms revealed that $92 \%$ isolates were positive for fimC gene, $80 \%$ for $t$ sh gene, $72 \%$ for $i u c D, 60 \%$ for fyuA and $48 \%$ for papC and $32 \%$ for irp2 (Table 3).

Table 3. The frequency of six genes studied in $E$. coli from field cases is enlisted.

\begin{tabular}{lcccc}
\hline Gene & Frequency & Farms & Percentage & C.I. \\
\hline fimC & 46 & 23 & 92 & 81.82 to \\
& & & & 97.41 \\
Tsh & 40 & 20 & 80 & 67.24 to \\
& & & & 89.37 \\
iucD & 36 & 18 & 72 & 58.42 to \\
& & & & 83.09 \\
fyuA & 30 & 15 & 60 & 46.04 to \\
& & & & 72.83 \\
papC & 24 & 12 & 48 & 34.46 to \\
& & & & 61.76 \\
Irp2 & 16 & 8 & 32 & 20.23 to \\
& & & & 45.80 \\
\hline
\end{tabular}

A total of 17 strains were identified on the basis of presence or absence of six genes (Table 4). The strains at each farm were, however, the same isolated from two selected birds on that farm. One strain was present on five farms having no papC and irp2 genes, another strain was isolated from two farms without irp2 gene. A strain isolated from two other farms was deficient in $f y u A$ gene, while a strain isolated from another farm was deficient in iucD and irp2 genes. Similarly, a strain isolated from two farms was deficient in $t s h$, papC and irp 2 genes. Other all strains $(n=12)$ were isolated from independent single farms with missing of single of multiple genes in different combinations (Table 4).
Table 4. Strains identified on the basis of presence or absence of six genes.

\begin{tabular}{lcl}
\hline Strain & Number of farms & Missing genes \\
\hline ECS1 & 5 & papC, Irp2 \\
ECS2 & 2 & Irp2 \\
ECS3 & 2 & fyuA \\
ECS4 & 2 & iucD, papC \\
ECS5 & 2 & Tsh, papC, Irp2 \\
ECS6 & 1 & iucD \\
ECS7 & 1 & iucD, Irp2 \\
ECS8 & 1 & iucD, fyuA \\
ECS9 & 1 & fyuA, Irp2 \\
ECS10 & 1 & fyuA, papC \\
ECS11 & 1 & fyuA, papC, Irp2 \\
ECS12 & 1 & iucD, fyuA, Irp2 \\
ECS13 & 1 & fimC, Irp2 \\
ECS14 & 1 & fimC, Tsh \\
ECS15 & 1 & Tsh, fyuA, Irp2, \\
ECS16 & 1 & Tsh, fyuA, papC, Irp2, \\
ECS17 & 1 & iucD, fyuA, papC, Irp2, \\
\hline
\end{tabular}

Pathogenicity genes and microscopic lesions: The fimC gene was detected in $64 \%$ cases, while $t s h$ and $i u c D$ genes in $56 \%$ cases each showing severe microscopic changes in the liver (Table 5). The fyuA and papC genes were detected in $36 \%$ cases each, while irp 2 gene was detected in $28 \%$ cases showing severe microscopic changes in liver. The genes fimC, tsh, iucD, fyuA, papC and irp2 were not detected in 8, 25, 28, 40,52 and $68 \%$ cases, respectively though microscopic changes of varying degree were seen in the liver.

The fim $C$ gene was detected in $56 \%$ cases, while $t s h$ and iucD genes were detected in $48 \%$ and $44 \%$ cases, respectively showing severe microscopic changes in heart (Table 5). The fyuA and papC genes were detected in $36 \%$ cases each, while irp 2 gene was detected in $28 \%$ cases showing severe microscopic changes. The genes fimC, tsh, iucD, fyuA, papC and $\operatorname{irp} 2$ were not detected in 8, 25, 28, 40, 52 and 72\% cases, respectively though microscopic changes of varying degree (mild to moderate) were seen in the heart.

The fimC, tsh, iucD, fyuA, papC and irp2 genes were detected in $24,16,20,4,16$ and $28 \%$ cases, respectively showing severe microscopic changes in spleen (Table 5). The genes fim $C, t s h, i u c D$, fyuA, papC and irp2 were not detected in 8, $25,28,40,52$ and $72 \%$ cases, respectively though microscopic changes of varying degree were seen in the spleen.

The fimC, tsh, iucD, fyuA, papC and irp2 genes were detected in 24, 16 and 20, 4, 16 and $28 \%$ cases, respectively showing severe microscopic changes in the intestine (Table 5). The fim $C$, tsh, iucD, fyuA, papC and irp 2 genes were not detected in $8,25,28,40,52$ and $72 \%$ cases, respectively though microscopic changes of varying degree were seen in the intestine. 
Table 5. Association of fimC,tsh, iucD, fyuA, papC and irp2 genes with severity of microscopic lesions in liver, heart, spleen and intestine.

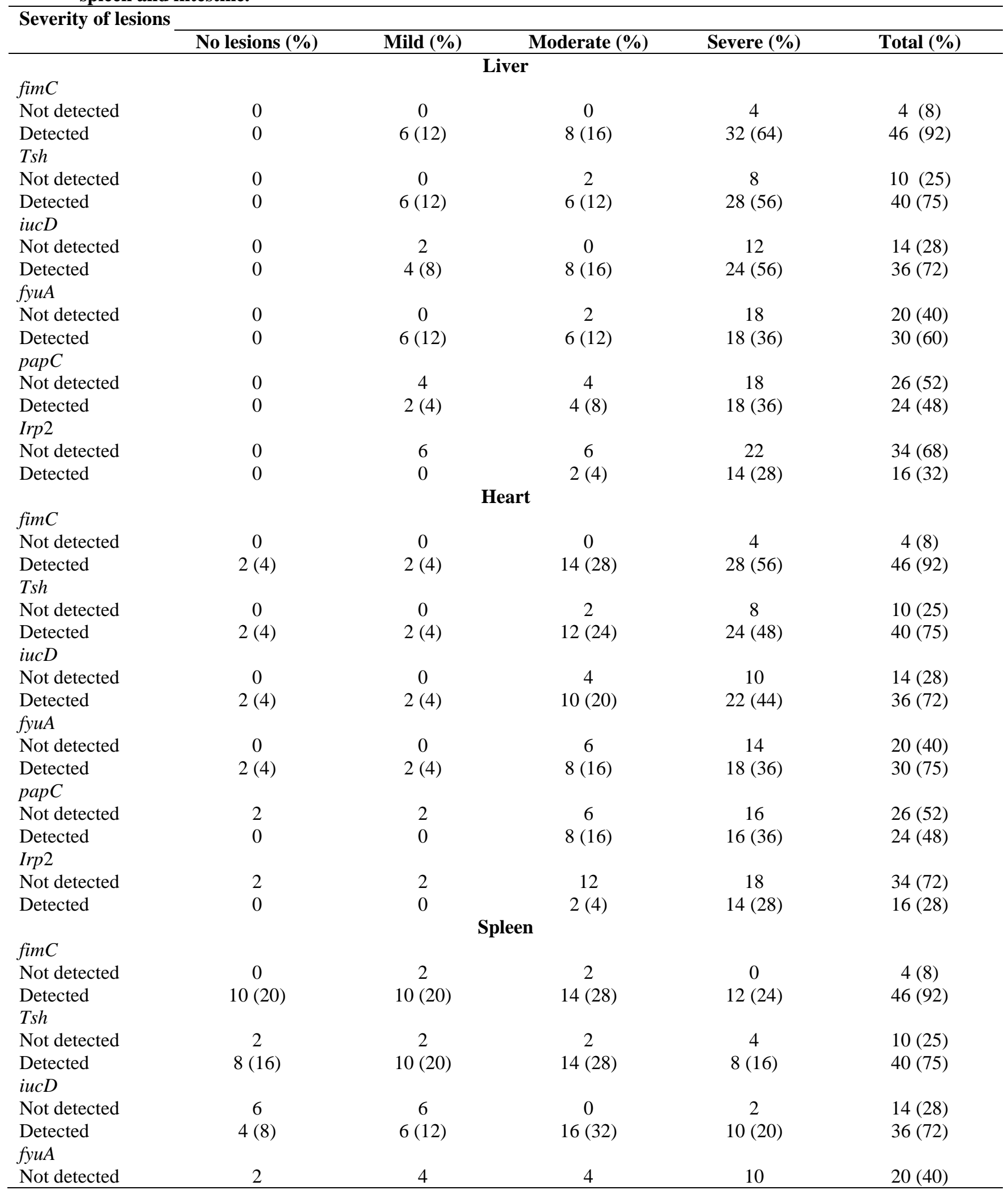




\begin{tabular}{|c|c|c|c|c|c|}
\hline \multirow{2}{*}{\multicolumn{6}{|c|}{ Severity of lesions }} \\
\hline & No lesions (\%) & Mild (\%) & Moderate (\%) & Severe $(\%)$ & Total (\%) \\
\hline $\begin{array}{l}\text { Detected } \\
\text { papC }\end{array}$ & $8(16)$ & $8(16)$ & $12(24)$ & $2(4)$ & $30(60)$ \\
\hline Not detected & 8 & 6 & 8 & 4 & $26(52)$ \\
\hline Detected & $2(4)$ & $6(12)$ & $8(16)$ & $8(16)$ & $24(48)$ \\
\hline $\operatorname{Irp2}$ & & & & & \\
\hline Not detected & 6 & 8 & 14 & 6 & $34(72)$ \\
\hline Detected & $4(8)$ & $4(8)$ & $2(4)$ & $6(12)$ & $16(28)$ \\
\hline \multicolumn{6}{|c|}{ Intestine } \\
\hline $\operatorname{fim} C$ & & & & & \\
\hline Not detected & 0 & 0 & 2 & 2 & $4(8)$ \\
\hline Detected & $2(4)$ & $8(16)$ & $18(36)$ & $18(36)$ & $46(92)$ \\
\hline Tsh & & & & & \\
\hline Not detected & 0 & 0 & 6 & 4 & $10(25)$ \\
\hline $\begin{array}{l}\text { Detected } \\
\text { iucD }\end{array}$ & $2(4)$ & $8(16)$ & $14(28)$ & $16(32)$ & $40(75)$ \\
\hline Not detected & 0 & 4 & 4 & 6 & $14(28)$ \\
\hline $\begin{array}{l}\text { Detected } \\
\text { fyuA }\end{array}$ & $2(4)$ & $4(8)$ & $16(32)$ & $14(28)$ & $36(72)$ \\
\hline Not detected & 0 & 4 & 10 & 6 & $20(40)$ \\
\hline $\begin{array}{l}\text { Detected } \\
\text { papC }\end{array}$ & $2(4)$ & $4(8)$ & $10(20)$ & $14(28)$ & $30(60)$ \\
\hline Not detected & 2 & 4 & 10 & 10 & $26(52)$ \\
\hline $\begin{array}{l}\text { Detected } \\
\text { Irp2 }\end{array}$ & 0 & $4(8)$ & $10(20)$ & $10(20)$ & $24(48)$ \\
\hline Not detected & 2 & 4 & 12 & 16 & $34(72)$ \\
\hline Detected & 0 & $4(8)$ & $8(16)$ & $4(8)$ & $16(28)$ \\
\hline
\end{tabular}

\section{DISCUSSION}

Colibacillosis is one of the important diseases of poultry, which causes lesions in different tissues of the body. The disease is caused by avian pathogenic Escherichia coli. It produces lesions such as air sacculitis, peritonitis, perihepatitis, pericarditis, osteomyelitis, synovitis or omphalitis (Kumar et al., 2013). Necropsy findings during the present study revealed lesions in different body tissues of broilers but the most affected organs were the liver and heart showing the fibrin layer on their surface and congestion. liver samples from $E$. coli infected birds showed $12 \%$ mild, $16 \%$ moderate and $72 \%$ severe microscopic lesions. Heart showed $8 \%$ mild, $40 \%$ moderate and $52 \%$ severe lesions. Spleen showed $44 \%$ mild, $12 \%$ moderate and $24 \%$ severe lesions and intestine showed $20 \%$ mild, $48 \%$ moderate and $28 \%$ severe histopathological lesions.

Gross and microscopic changes in different organs observed in present study corresponded with the findings of others (Ghosh et al., 2006; Kumar et al., 2013). However, during present study, some differences were also observed in gross and microscopic changes. In $4 \%$ cases, the moderate gross lesion recorded in liver were confirmed as severe lesions under the microscope. There may be a judgment difference in recording the gross changes and sometime the lesion observed grossly are less visible as they are present in severe degree deeper in the tissues. Similarly, in $12 \%$ cases, the moderate gross lesions recorded grossly in heart were confirmed as severe lesions under the microscope. For spleen, 20\% lesions recorded as mild grossly were confirmed as moderate lesions under microscope. For intestine, 4\% lesions recorded grossly as mild were confirmed as moderate under the microscope, while in $12 \%$ cases, moderate lesions recorded grossly were confirmed as severe lesions under the microscope. These results suggest that the microscopic evaluation of tissue for pathological change discerns better information and there are always differences in recognizing and categorizing lesions on the basis of gross observations and sometimes more severe microscopic changes are present in deeper areas of tissues showing only mild or moderate gross lesions. Therefore, we considered histopathological changes, while looking for association of genes in isolates and the effects on various tissues.

Pathogenicity genes and microscopic lesions: In the present study, 6 virulent genes were targeted which were reported in most of the studies, which are typically associated with APEC and they are grouped into 3 classes; genes essential for adhesion ( $p a p C$ and fim $C$ ), serum resistance ( $t s h$ ), iron uptake (irp2 and iucD) and development of lesions and fibrin deposit in the air sacs $(f y u A)$ (Ewers et al., 2004). The fimC is an 
important gene having the role in the internal organ adhesion (Jan Ben et al., 2001). This gene was detected in $92 \%$ cases. According to the present study, $80 \%$ of the isolates were positive for $t s h$ gene. The genes iucD, fyuA, papC and irp2 were detected at the rate of $72 \%, 60 \%, 48 \%$ and $32 \%$, respectively. Earlier it has been concluded that fim $C$ gene was present with the highest rate, i.e., $92 \%$, while $i u c D, t s h, f y u A$, irp2 and papC were present in the $88.7 \%, 85.3 \%, 66 \%, 68 \%$ and $30 \%$ cases, respectively (JanBen et al., 2001). Previously from a study on 16 virulent genes from 200 isolates of colibacillosis in Brazil by PCR, seven genes (tsh, papC, papG, iss, cvaC and felA) were detected with highest frequency and confirmed that these genes have major roles in the pathogenesis of colibacillosis (Delicato et al., 2003). Another study concluded that fimC was present in $96 \%$ cases, followed by papC (16\%) and sfa (6\%) (Knobl et al., 2004). Similarly, another study detected genes with highest proportion were iss $(73.8 \%)$, tsh $(55.7 \%)$ and papC $(24.3 \%)$ (Rocha et al., 2008). Another study reported that the genes detected most frequently from $E$. coli isolates were fim $C$ with a ratio of $93.6 \%$, followed by $i u c D$, iss, and $t s h$, i.e. $70.8 \%$, $58.8 \%$ and $51.4 \%$, respectively (Wen-Ji et al. 2008). It has been reported that the omp $T$ genes was detected with highest rate $(94.1 \%)$, followed by fimC $(90.1 \%)$, and $h l y F(87.1 \%)$ (Jeong et al., 2012). Other genes they detected included iucD, tsh, fyuA and irp2with the following rate of $73.3 \%, 61.4 \%$, $44.6 \%$ and $43.6 \%$, respectively. These results indicated the difference in types of genes and their frequency from $E$. coli in different parts of the world, but such a data is needed to have an understanding as what kind of strains are present in different parts of the world.

The results about presence of genes and severity of lesions from field cases suggests their association with the lesions produced, however, the experimental studies are required to confirm these findings. In field cases, different birds might be having a difference in stage of disease, where changes might not have been advanced due to time factor or it may be otherwise. However, these results do indicate the involvement of certain genes more than others in producing severe changes in different body tissues as in more than $50 \%$ cases or close, the genes including fim $C, T s h$ and $i u c D$ were detected from cases with severe changes in the liver and more or less in the heart. A study concluded from their findings that among $t s h$ positive $E$. coli isolates, $90.6 \%$ belonged to highest virulence class (Dozois et al., 2000). A study was carried out on nine virulence associated genes in E. coli and relationship of these genes to the chicken embryo lethality (Oh et al., 2012). They observed those genes with following ranked order, fimC (93.1\%), iucD (67.24\%), followed by iss (58.62\%), tsh (34.48\%) and fyuA (32.76\%). They further reported that these virulent genes had direct relationship with embryo mortality. From these results, it can be said that there are a number of genes those are involved in different combinations to produce lesions in different body organs of the chicken in different parts of the world (Oh et al., 2012). Therefore, more studies are required to understand these genes from different strains as for frequency and lethality is concerned that will help to devise better control measures.

Conclusions: With the help of PCR for detection of pathogenicity related genes it is possible to not only detect these genes, but also can be used for strain typing. There is a positive association between pathogenicity genes of $E$. coli with gross and histopathological lesions in the liver, heart, spleen and intestine.

Competing interests: The authors declare that they have no competing interests.

Acknowledgements: The laboratory staff of Department of Pathology, Faculty of Veterinary Science, University of Agriculture Faisalabad is gratefully acknowledged.

\section{REFERENCES}

Ahmad, I., M.S. Anjum and M. Hanif. 2012. Prevalence of poultry diseases at high altitudes of District Poonch Azad Jammu and Kashmir. Pak. J. Sci. 64:334-336.

Alexander, D.J. 2000. The history of avian influenza in poultry. World Poult. Elsevier special, USA. Pp. 7-8.

Anjum, A.D. 1997. Poultry diseases. $2^{\text {nd }}$ Ed. Vet Ag publications, Pakistan. pp: 105-110.

Bancroft, J.D. and M. Gamble. 2008. Theory and Practice of Histopthological Technique. $5^{\text {th }}$ Ed. Churchill Livingston London. pp:85-100.

Bhalerao, A.K.D., R.P. Gupta and M. Kumari. 2013. Pathological studies on natural cases of avian Colibacillosis in Haryana state. Haryana Vet. 52:118120.

Biswas, P.K., G.M.N. Uddin, H. Barua, K. Roy, D. Biswas, A. Ahad and N.C. Debnath. 2006. Causes of loss of sonali chickens on smallholder households in Bangladesh. Prev. Vet. Med. 76:185-195.

Carter, G.R. 1986. Essentials of Veterinary Bacteriology and Mycology. $3^{\text {rd }}$ Ed. Lippincott Williams and Wikins, USA. pp. 312-330.

Delicato, E.R., B.G.D. Brito, L.C.J. Gaziri and M.C. Vidotto. 2003. Virulence-associated genes in Escherichia coli isolates from poultry. Vet. Microb. 94:97-103.

Dozois, C.M., M. Dhu-Moulin, E. Anniebree, J.M. Faitbrother, C. Desautels and R. Curtiss. 2000. Relationship between the $t s h$ autotransporter and pathogenicity of avian Escherichia coli and localization and analysis of the $t s h$ genetic region. Infect Immun. 68:4145-4158.

Ewers, C., T. JanBenm, S. KieBling, H.C. Philipp and L.H. Wieler. 2004. Molecular epidemiology of avian 
pathogenic Escherichia coli (APEC) isolated from colisepticemia in poultry. Vet Microb. 104:91-101.

Ghosh, R.C., S.D. Hirpurkar and P.R. Suryawnshi. 2006. Concurrent colibacillosis and infectious bursal disease in broiler chicks. Indian Vet. J. 83:1019-1020.

Gomes, A.R., L. Muniyapz, G. Krishnappa, V.V.S. Suryanarayana, S. Isloor, B. Prakash and P.G. Hugar. 2005. Genotypic characterization of avian Escherichia coli by random amplification of polymorphic DNA. Int. J. Poult. Sci. 4:378-381.

JanBen, T., C. Schwarzi, P. Preikschati, M. Voss, H.C. Philipp and L.H. Wieler. 2001. Virulence-associated genes in avian pathogenic Escherichia coli (APEC) isolated from internal organs of poultry having died from colibacillosis. Int. J. Med. Microb. 291:371-378.

Jeong, Y.W., T.E. Kim, J.H. Kim and H.J. Kwon. 2012. Pathotyping avian pathogenic Escherichia coli strains in Korea. J. Vet. Sci. 13:145-152.

Knobl, T., T.A.T. Gomes, M.A.M. Vieira, J.A. Bottinoa and A.J.P. Ferreira. 2004. Detection of pap, sfa, afa and fimc adhesin-encoding operons in avian pathogenic Escherichia coli. Int. J. Appl. Res. Vet. Med. 2:135-141.

Mazaheri, S., S.S. Ahrabi and M.M. Aslani. 2014. Shiga toxin-producing Escherichia coli isolated from lettuce samples in Tehran, Iran. Jundishapur J. Microbiol. 7:12346.

Oh, J.Y., M.S. Kang, H. Yoon, H.W. Choi, B.K. An, E.G. Shin, Y.J. Kim, M.J. Kim, J.H. Kwon and Y.K. Kwon. 2012. The embryo lethality of Escherichia coli isolates and its relationship to the presence of virulenceassociated genes. Poult Sci. 91:370-375.

Rocha, A.C.G.P., S.L.S. Rocha, C.A.V.L. Rosa, G.F. Souza, H.L.S. Moraes, F.O. Salle, L.B. Moraes and C.T.P. Salle. 2008. Genes associated with pathogenicity of avian Escherichia coli (APEC) isolated from respiratory cases of poultry. Pesqu Vet. Brasil. 28:183-186.

Rodriguez-Siek, K.E., C.W. Giddings, C. Doetkott, T.J. Johnson and L.K. Nolan. 2005. Characterizing the APEC pathotype. Vet. Res. 36:241-256.

Tonu, N.S., M.A. Sufian, S. Sarker, M.M. Kamal, M.H. Rahman and M.M. Hossain. 2011. Pathological study on colibacillosis in chickens and detection of Escherichia coli by PCR. Bangladesh J. Vet. Med. 9:17- 25.

Wen-ji, J., Z. Zhi-ming, Z. Yong-zhi, Q. Ai-jian, S. Hong-xia, L.I.U. Yue-long, W. Jiao and W.Q. Qian. 2008. Distribution of virulence-associated genes of avian pathogenic Escherichia coli isolates in china. Agri. Sci. China. 7:1511-1515. 